\title{
ON EQUAL VALUES OF POWER SUMS OF ARITHMETIC PROGRESSIONS
}

\author{
András Bazsó, Dijana Kreso, Florian luca and Ákos Pintér
}

University of Debrecen, Hungary, Technische Universität Graz, Austria and Universidad Nacional Autónoma de México, Mexico

ABStRACT. In this paper, we consider the Diophantine equation

$$
\begin{aligned}
& b^{k}+(a+b)^{k}+\cdots+(a(x-1)+b)^{k}= \\
& =d^{l}+(c+d)^{l}+\cdots+(c(y-1)+d)^{l},
\end{aligned}
$$

where $a, b, c, d, k, l$ are given integers with $\operatorname{gcd}(a, b)=\operatorname{gcd}(c, d)=1, k \neq l$.

We prove that, under some reasonable assumptions, the above equation has only finitely many solutions.

\section{INTRODUCTION AND RESULTS}

For a positive integer $n \geq 2$, let

$$
S_{a, b}^{k}(n)=b^{k}+(a+b)^{k}+\cdots+(a(n-1)+b)^{k} .
$$

It is easy to see that the above power sum is related to the Bernoulli polynomials $B_{k}(x)$ in the following way:

$$
\begin{aligned}
S_{a, b}^{k}(n)= & \frac{a^{k}}{k+1}\left(\left[B_{k+1}\left(n+\frac{b}{a}\right)-B_{k+1}\right]\right. \\
& \left.-\left[B_{k+1}\left(\frac{b}{a}\right)-B_{k+1}\right]\right),
\end{aligned}
$$

where the polynomials $B_{k}(x)$ is defined by the generating series

$$
\frac{t \exp (t x)}{\exp (t)-1}=\sum_{k=0}^{\infty} B_{k}(x) \frac{t^{k}}{k !}
$$

2010 Mathematics Subject Classification. 11B68, 11D41.

Key words and phrases. Diophantine equations, exponential equations, Bernoulli polynomials. 
and $B_{k+1}=B_{k+1}(0)$. For the properties of Bernoulli polynomials which will be often used in this paper, sometimes without special reference, we refer to [7, Chapters 1 and 2]. We can extend $S_{a, b}^{k}$ for every real value $x$ as

$$
S_{a, b}^{k}(x)=\frac{a^{k}}{k+1}\left(B_{k+1}\left(x+\frac{b}{a}\right)-B_{k+1}\left(\frac{b}{a}\right)\right) .
$$

We denote by $\mathbb{C}[x]$ the ring of polynomials in the variable $x$ with complex coefficients. A decomposition of a polynomial $F(x) \in \mathbb{C}[x]$ is an equality of the following form

$$
F(x)=G_{1}\left(G_{2}(x)\right) \quad\left(G_{1}(x), G_{2}(x) \in \mathbb{C}[x]\right),
$$

which is nontrivial if

$$
\operatorname{deg} G_{1}(x)>1 \quad \text { and } \quad \operatorname{deg} G_{2}(x)>1 .
$$

Two decompositions $F(x)=G_{1}\left(G_{2}(x)\right)$ and $F(x)=H_{1}\left(H_{2}(x)\right)$ are said to be equivalent if there exists a linear polynomial $\ell(x) \in \mathbb{C}[x]$ such that $G_{1}(x)=H_{1}(\ell(x))$ and $H_{2}(x)=\ell\left(G_{2}(x)\right)$. The polynomial $F(x)$ is called decomposable if it has at least one nontrivial decomposition; otherwise it is said to be indecomposable.

In a recent paper, Bazsó, Pintér and Srivastava ([1]) proved the following theorem about the decomposition of the polynomial $S_{a, b}^{k}(x)$ defined above.

TheOREM 1.1. The polynomial $S_{a, b}^{k}(x)$ is indecomposable for even $k$. If $k=2 v-1$ is odd, then any nontrivial decomposition of $S_{a, b}^{k}(x)$ is equivalent to the following decomposition:

$$
S_{a, b}^{k}(x)=\widehat{S}_{v}\left(\left(x+\frac{b}{a}-\frac{1}{2}\right)^{2}\right) .
$$

Proof. This is [1, Theorem 2].

Using Theorem 1.1 and the general finiteness criterion of Bilu and Tichy ([2]) for Diophantine equations of the form $f(x)=g(y)$, we prove the following result.

TheOrem 1.2. For $2 \leq k<l$, the equation

$$
S_{a, b}^{k}(x)=S_{c, d}^{l}(y)
$$

has only finitely many solutions in integers $x$ and $y$.

Since the finiteness criterion from [2] is based on the ineffective theorem of Siegel, our Theorem 1.2 is ineffective. We note that for $a=c=1, b=d=0$ our theorem gives the result of Bilu, Brindza, Kirschenhofer, Pintér and Tichy $([3])$.

Combining a result of Brindza [5] with recent theorems by Rakaczki ([8]) and Pintér and Rakaczki $([6])$, for $k=1$ and 3 we obtain effective statements. 
Theorem 1.3. For $k=1$ and $l \notin\{1,3,5\}$, the equation

$$
S_{a, b}^{1}(x)=S_{c, d}^{l}(y)
$$

implies $\max (|x|,|y|)<C_{1}$, where $C_{1}$ is an effectively computable constant depending only on $a, b, c, d$ and $l$.

In the exceptional cases $l=3,5$ one can give some values for $a, b, c, d$ such that the corresponding equations possess infinitely many solutions. For example, if $k=1, a=2, b=1, l=3$ or $l=5, c=1, d=0$ we have

$$
x^{2}=1+3+\cdots+2 x-1=1^{3}+2^{3}+\cdots+(y-1)^{3}
$$

or

$$
x^{2}=1+3+\cdots+2 x-1=1^{5}+2^{5}+\cdots+(y-1)^{5},
$$

respectively. These equations have infinitely many integer solutions, see [9].

Theorem 1.4. For $k=3$ and $l \notin\{1,3,5\}$, the equation

$$
S_{a, b}^{3}(x)=S_{c, d}^{l}(y)
$$

implies $\max (|x|,|y|)<C_{2}$, where $C_{2}$ is an effectively computable constant depending only on $a, b, c, d$ and $l$.

\section{Auxiliary Results}

In this section, we collect some results needed to prove Theorem 1.2. First, we recall the finiteness criterion of Bilu and Tichy ([2]). To do this, we need to define five kinds of so-called standard pairs of polynomials.

Let $\alpha, \beta$ be nonzero rational numbers, $\mu, \nu, q>0$ and $\rho \geq 0$ be integers, and let $\nu(x) \in \mathbb{Q}[x]$ be a nonzero polynomial (which may be constant).

A standard pair of the first kind is $\left(x^{q}, \alpha x^{\rho} \nu(x)^{q}\right)$ or switched, $\left(\alpha x^{\rho} \nu(x)^{q}\right.$, $\left.x^{q}\right)$, where $0 \leq \rho<q, \operatorname{gcd}(\rho, q)=1$ and $\rho+\operatorname{deg} \nu(x)>0$.

A standard pair of the second kind is $\left(x^{2},\left(\alpha x^{2}+\beta\right) \nu(x)^{2}\right)$ or switched.

Denote by $D_{\mu}(x, \delta)$ the $\mu$-th Dickson polynomial, defined by the functional equation

$$
D_{\mu}(z+\delta / z, \delta)=z^{\mu}+(\delta / z)^{\mu}
$$

or by the explicit formula

$$
D_{\mu}(x, \delta)=\sum_{i=0}^{\lfloor\mu / 2\rfloor} d_{\mu, i} x^{\mu-2 i} \quad \text { with } \quad d_{\mu, i}=\frac{\mu}{\mu-i}\left(\begin{array}{c}
\mu-i \\
i
\end{array}\right)(-\delta)^{i} .
$$

A standard pair of the third kind is $\left(D_{\mu}\left(x, \alpha^{\nu}\right), D_{\nu}\left(x, \alpha^{\mu}\right)\right)$, where $\operatorname{gcd}(\mu, \nu)=1$.

A standard pair of the fourth kind is

$$
\left(\alpha^{-\mu / 2} D_{\mu}(x, \alpha),-\beta^{-\nu / 2} D_{\nu}(x, \beta)\right),
$$

where $\operatorname{gcd}(\mu, \nu)=2$.

A standard pair of the fifth kind is $\left(\left(\alpha x^{2}-1\right)^{3}, 3 x^{4}-4 x^{3}\right)$ or switched. 
The following theorem is the main result of [2].

TheOREM 2.1. Let $R(x), S(x) \in \mathbb{Q}[x]$ be nonconstant polynomials such that the equation $R(x)=S(y)$ has infinitely many solutions in rational integers $x, y$. Then $R=\varphi \circ f \circ \kappa$ and $S=\varphi \circ g \circ \lambda$, where $\kappa(x), \lambda(x) \in \mathbb{Q}[x]$ are linear polynomials, $\varphi(x) \in \mathbb{Q}[x]$, and $(f(x), g(x))$ is a standard pair.

The following lemmas are the main ingredients for the proofs of Theorems 1.3 and 1.4.

Lemma 2.2. For every $b \in \mathbb{Q}$ and rational integer $k \geq 3$ with $k \notin\{4,6\}$ the polynomial $B_{k}(x)+b$ has at least three zeros of odd muliplicities.

Proof. For $b=0$ and odd values of $k \geq 3$ this result is a consequence of a theorem by Brillhart ([4, Corollary of Theorem 6$])$. For non-zero rational $b$ and odd $k$ with $k \geq 3$ and for even values of $k \geq 8$ our lemma follows from $[6$, Theorem] and $[8$, Theorem 2. 3], respectively.

Our next auxiliary result is an easy consequence of an effective theorem concerning the $S$-integer solutions of so-called hyperelliptic equations.

LEMma 2.3. Let $f(x)$ be a polynomial with rational coefficients and with at least three zeros of odd multiplicities. Further, let $u$ be a fixed positive integer. If $x$ and $y$ are integer solutions of the equation

$$
f\left(\frac{x}{u}\right)=y^{2}
$$

then we have $\max (|x|,|y|)<C_{3}$, where $C_{3}$ is an effectively computable constant depending only on $u$ and the parameters of $f$.

Proof. This is a special case of the main result of [5].

Let $c_{1}, e_{1} \in \mathbb{Q}^{*}$ and $c_{0}, e_{0} \in \mathbb{Q}$.

Lemma 2.4. The polynomial $S_{a, b}^{k}\left(c_{1} x+c_{0}\right)$ is not of the form $e_{1} x^{q}+e_{0}$ with $q \geq 3$.

LEMma 2.5. The polynomial $S_{a, b}^{k}\left(c_{1} x+c_{0}\right)$ is not of the form

$$
e_{1} D_{\nu}(x, \delta)+e_{0},
$$

where $D_{\nu}(x, \delta)$ is the $\nu$-th Dickson polynomial with $\nu>4, \delta \in \mathbb{Q}^{*}$.

Before proving the above lemmas, we introduce the following notation. Put

$$
S_{a, b}^{k}\left(c_{1} x+c_{0}\right)=s_{k+1} x^{k+1}+s_{k} x^{k}+\cdots+s_{0}
$$

and

$$
c_{0}^{\prime}=\frac{b}{a}+c_{0}
$$


We have

$$
\begin{aligned}
s_{k+1} & =\frac{a^{k} c_{1}^{k+1}}{k+1}, \\
s_{k} & =\frac{a^{k} c_{1}^{k}}{2}\left(2 c_{0}^{\prime}-1\right), \\
s_{k-1} & =\frac{a^{k} c_{1}^{k-1}}{12} k\left(6 c_{0}^{\prime 2}-6 c_{0}^{\prime}+1\right), k \geq 2,
\end{aligned}
$$

and for $k \geq 4$,

$$
s_{k-3}=\frac{a^{k} c_{1}^{k-3}}{720} k(k-1)(k-2)\left(30 c_{0}^{\prime 4}-60 c_{0}^{\prime 3}+30 c_{0}^{\prime 2}-1\right) .
$$

Proof of Lemma 2.4. Suppose that $S_{a, b}^{k}\left(c_{1} x+c_{0}\right)=e_{1} x^{q}+e_{0}$, where we have $q=k+1 \geq 3$. It follows that $s_{k-1}=0$, so $6 c_{0}^{\prime 2}-6 c_{0}^{\prime}+1=0$. Hence, $c_{0}^{\prime} \notin \mathbb{Q}$, which is a contradiction.

Proof of Lemma 2.5. Suppose that $S_{a, b}^{k}\left(c_{1} x+c_{0}\right)=e_{1} D_{\nu}(x, \delta)+e_{0}$ with $\nu>4$. Then

$$
\begin{aligned}
s_{k+1} & =e_{1}, \\
s_{k} & =0, \\
s_{k-1} & =-e_{1} \nu \delta, \\
s_{k-3} & =\frac{e_{1}(\nu-3) \nu \delta^{2}}{2} .
\end{aligned}
$$

From (2.8), (2.12) and (2.9), (2.13), respectively, it follows that

$$
e_{1}=\frac{a^{\nu-1} c_{1}^{\nu}}{\nu} \text { and } c_{0}^{\prime}=\frac{1}{2} \text {. }
$$

In view of (2.10), substituting (2.16) together with $k=\nu-1$ into (2.14), we obtain

$$
-\frac{a^{\nu-1} c_{1}^{\nu-2}(\nu-1)}{24}=-\frac{a^{\nu-1} c_{1}^{\nu} \nu \delta}{\nu}
$$

which implies

$$
c_{1}^{2}=\frac{\nu-1}{24 \delta} .
$$

Similarly, comparing the forms (2.11) and (2.15) of $s_{k-3}$ with the substitutions $k=\nu-1$ and (2.16), we obtain

$$
\frac{7 a^{\nu-1} c_{1}^{\nu-4}(\nu-1)(\nu-2)(\nu-3)}{5760}=\frac{a^{\nu-1} c_{1}^{\nu}(\nu-3) \nu \delta^{2}}{2 \nu}
$$

which implies

$$
c_{1}^{4}=\frac{7(\nu-1)(\nu-2)}{2880 \delta^{2}} .
$$


After substituting (2.18) into (2.20), we obtain $7(\nu-2)=5(\nu-1)$, which implies $\nu=9 / 2$, a contradiction.

One can see that the condition $\nu>4$ is necessary. Indeed,

$$
S_{2,1}^{2}(x)=\frac{4}{3} x^{3}-\frac{1}{3} x=\frac{4}{3} D_{3}\left(x, \frac{1}{12}\right),
$$

and

$$
S_{2,1}^{3}(x)=2 x^{4}-x^{2}=2 D_{4}\left(x, \frac{1}{8}\right)-\frac{1}{16} .
$$

\section{Proofs of the Theorems}

Proof of Theorem 1.3. Using (3), one can rewrite equation (6) as

$$
\frac{c^{l}}{l+1}\left(B_{l+1}\left(y+\frac{d}{c}\right)-B_{l+1}\left(\frac{d}{c}\right)\right)=\frac{1}{2} a x^{2}+\left(b-\frac{a}{2}\right) x
$$

or

$$
\begin{aligned}
\frac{8 a c^{l}}{l+1}\left(B_{l+1}\left(y+\frac{d}{c}\right)-B_{l+1}\left(\frac{d}{c}\right)\right) & =4 a^{2} x^{2}+8 a\left(b-\frac{a}{2}\right) x \\
& =(2 a x+2 b-a)^{2}-(2 b-a)^{2} .
\end{aligned}
$$

Then our result is a simple consequence of Lemmas 2.2 and 2.3.

Proof of Theorem 1.3. Following Theorem 1.1, we have

$$
\begin{aligned}
S_{a, b}^{3}(x)= & \frac{a^{3}}{4}\left(x+\frac{b}{a}-\frac{1}{2}\right)^{4}-\frac{a^{3}}{8}\left(x+\frac{b}{a}-\frac{1}{2}\right)^{2} \\
& +\frac{a^{4}-16 a^{2} b^{2}+32 a b^{3}-16 b^{4}}{64 a} .
\end{aligned}
$$

Using the above representation, we rewrite equation (7) as

$64 a S_{c, d}^{l}(y)=(2 a x+2 b-a)^{4}-4 a^{2}(2 a x+2 b-a)^{2}+a^{4}-16 a^{2} b^{2}+32 a b^{3}-16 b^{4}$

or

$$
64 a S_{c, d}^{l}(y)+3 a^{4}+16 a^{2} b^{2}-32 a b^{3}-16 b^{4}=\left(X-2 a^{2}\right)^{2},
$$

where $X=(2 a x+2 b-a)^{2}$. As in the previous case, Lemmas 2.2 and 2.3 complete the proof.

Proof of THEOREM 1.2. If the equation (5) has infinitely many integer solutions, then by Theorem 2.1 it follows that $S_{a, b}^{k}\left(a_{1} x+a_{0}\right)=\varphi(f(x))$ and $S_{c, d}^{l}\left(b_{1} x+b_{0}\right)=\varphi(g(x))$, where $(f, g)$ is a standard pair over $\mathbb{Q}, a_{0}, a_{1}, b_{0}, b_{1}$ are rationals with $a_{1} b_{1} \neq 0$ and $\varphi(x)$ is a polynomial with rational coefficients.

Assume that $h=\operatorname{deg} \varphi>1$. Then Theorem 1.1 implies

$$
0<\operatorname{deg} f, \operatorname{deg} g \leq 2,
$$


and since $k<l$, we have $\operatorname{deg} f=1, \operatorname{deg} g=2$. In particular, $k+1=h$ and $l+1=2 h$, so $l=2 k+1$. Therefore, if $l \neq k+1$, we then must have $h=\operatorname{deg} \varphi=1$ and $l=2 k+1$.

Condition $k \neq 1$ implies $k \geq 2$ and since $l=2 k+1$, it follows that $l \geq 5$. Since $\operatorname{deg} f=1$, there exist $f_{1}, f_{0} \in \mathbb{Q}, f_{1} \neq 0$, such that $S_{a, b}^{k}\left(f_{1} x+f_{0}\right)=\varphi(x)$, so

$$
S_{a, b}^{k}\left(f_{1} g(x)+f_{0}\right)=\varphi(g(x))=S_{c, d}^{l}\left(b_{1} x+b_{0}\right) .
$$

As $g(x)$ is quadratic, by making the substitution $x \mapsto\left(x-b_{0}\right) / b_{1}$, we obtain that there are $c_{2}, c_{1}, c_{0} \in \mathbb{Q}, c_{2} \neq 0$, such that

$$
S_{a, b}^{k}\left(c_{2} x^{2}+c_{1} x+c_{0}\right)=S_{c, d}^{l}(x) .
$$

Since $\operatorname{deg} S_{a, b}^{k}(x)=k+1 \geq 2$ and $c_{2} \neq 0$, we have a decomposition of $S_{c, d}^{l}(x)$ which is equivalent to $S\left((x+b / a-1 / 2)^{2}\right)$ for some $S \in \mathbb{Q}[x]$ with $\operatorname{deg} S=k+1$, according to Theorem 1.1. Therefore, there exists a linear polynomial $l(x)$ in $\mathbb{C}[x]$ such that

$$
c_{2} x^{2}+c_{1} x+c_{0}=l\left((x+b / a-1 / 2)^{2}\right)
$$

and $S(x)=S_{a, b}^{k}(l(x))$. Hence, there are $A, B \in \mathbb{C}, A \neq 0$, such that

$$
c_{2} x^{2}+c_{1} x+c_{0}=A(x+b / a-1 / 2)^{2}+B .
$$

Clearly, this implies that $A, B \in \mathbb{Q}$ and

$$
S_{a, b}^{k}\left(A(x+b / a-1 / 2)^{2}+B\right)=S_{c, d}^{2 k+1}(x) .
$$

By the linear substitution $x \mapsto x-b / a+1 / 2$, we obtain

$$
S_{a, b}^{k}\left(A x^{2}+B\right)=S_{c, d}^{2 k+1}(x-b / a+1 / 2) .
$$

Thus, we have an equality of polynomials of degree $2 k+2 \geq 6$. We calculate and compare coefficients of the first few highest monomials participating in the above polynomials. The coefficients of the polynomial in the right-hand side above are easily deduced by setting $c_{1}=1, c_{0}=-b / a+1 / 2$ in (2.8), (2.9), (2.10) and (2.11). Therefore, if we denote

$$
S_{c, d}^{2 k+1}(x-b / a+1 / 2)=r_{2 k+2} x^{2 k+2}+\cdots+r_{1} x+r_{0},
$$

and

$$
c_{0}^{\prime}=\frac{d}{c}-\frac{b}{a}+\frac{1}{2},
$$


then the coefficients are:

$$
\begin{aligned}
r_{2 k+2} & =\frac{c^{2 k+1}}{2 k+2}, \\
r_{2 k+1} & =\frac{c^{2 k+1}}{2}\left(2 c_{0}^{\prime}-1\right), \\
r_{2 k} & =\frac{c^{2 k+1}(2 k+1)}{12}\left(6 c_{0}^{\prime 2}-6 c_{0}^{\prime}+1\right), \\
r_{2 k-2} & =\frac{c^{2 k+1}(2 k+1) 2 k(2 k-1)}{720}\left(30 c_{0}^{\prime 4}-60 c_{0}^{\prime 3}+30 c_{0}^{\prime 2}-1\right) .
\end{aligned}
$$

On the other hand, the coefficients $s_{k+1}, s_{k}, \ldots s_{0}$ for the polynomial $S_{a, b}^{k}(x)$ can be found by setting $c_{1}=1, c_{0}=0$ in (2.8), (2.9), (2.10) and (2.11). Since

$$
S_{a, b}^{k}\left(A x^{2}+B\right)=\sum_{m=0}^{k+1} s_{m} \sum_{i=0}^{m}\left(\begin{array}{c}
m \\
i
\end{array}\right)\left(A x^{2}\right)^{i} B^{m-i},
$$

it follows that if we put

$$
S_{a, b}^{k}\left(A x^{2}+B\right)=t_{2 k+2} x^{2 k+2}+\cdots+t_{1} x+t_{0},
$$

then

$$
\begin{aligned}
t_{2 k+2}= & \frac{a^{k} A^{k+1}}{k+1} \\
t_{2 k+1}= & 0 \\
t_{2 k}= & a^{k} A^{k} B+\frac{a^{k} A^{k}}{2}\left(2\left(\frac{b}{a}\right)-1\right), \\
t_{2 k-1}= & 0 \\
t_{2 k-2}= & \frac{a^{k} k}{2} A^{k-1} B^{2}+\frac{a^{k} k}{2} A^{k-1} B\left(2\left(\frac{b}{a}\right)-1\right) \\
& +\frac{a^{k} k}{12} A^{k-1}\left(6\left(\frac{b}{a}\right)^{2}-6\left(\frac{b}{a}\right)+1\right) .
\end{aligned}
$$

Now we compare the coefficients. Comparing the leading coefficients yields

$$
\frac{a^{k} A^{k+1}}{k+1}=\frac{c^{2 k+1}}{2 k+2}, \quad \text { so } \quad 2 a^{k} A^{k+1}=c^{2 k+1},
$$

and

$$
\frac{2 c}{a}=\frac{c^{2 k+2}}{a^{k+1} A^{k+1}}
$$

Therefore,

$$
\sqrt[k+1]{\frac{2 c}{a}} \in \mathbb{Q}
$$


If $a$ and $c$ do not fulfill the above condition, we are through, otherwise we proceed. Comparing the coefficients of index $2 k+1$, we get

so $c_{0}^{\prime}=1 / 2$, which implies

$$
\frac{c^{2 k+1}}{2}\left(2 c_{0}^{\prime}-1\right)=0,
$$

$$
\frac{d}{c}=\frac{b}{a} .
$$

If the coefficients $a, b, c$ and $d$ do not satisfy the last property above, then we eliminate the possibility $\operatorname{deg} \varphi>1$. Therefore, we proceed with the case where $a, b, c$ and $d$ do satisfy this property. Comparing the next coefficients and using (3.22), we obtain

$$
\frac{b}{a}-\frac{1}{2}=-\frac{1}{12} A(2 k+1)-B .
$$

Comparing the coefficients of index $2 k-2$ and using $c_{0}^{\prime}=1 / 2$, we get

$$
\begin{aligned}
\frac{a^{k} k}{2} A^{k-1} B^{2} & +\frac{a^{k} k}{2} A^{k-1} B\left(2\left(\frac{b}{a}\right)-1\right) \\
& +\frac{a^{k} k}{12} A^{k-1}\left(6\left(\frac{b}{a}\right)^{2}-6\left(\frac{b}{a}\right)+1\right) \\
& =\frac{7}{8} \cdot \frac{c^{2 k+1}(2 k+1) 2 k(2 k-1)}{720} .
\end{aligned}
$$

By using also (3.22) and simplifying, we obtain

$$
\begin{aligned}
\frac{B^{2}}{2}+\frac{B}{2}\left(2\left(\frac{b}{a}\right)-1\right)+ & \frac{1}{12}\left(6\left(\frac{b}{a}\right)^{2}-6\left(\frac{b}{a}\right)+1\right) \\
& =\frac{7\left(4 k^{2}-1\right) A^{2}}{1440} .
\end{aligned}
$$

By using also (3.23), the last relation above can be transformed into

$$
\begin{aligned}
\frac{B^{2}}{2}+B\left(-\frac{1}{12} A(2 k+1)-B\right) & +\frac{1}{2}\left(-\frac{1}{12} A(2 k+1)-B\right)^{2}-\frac{1}{24} \\
& =\frac{7 A^{2}\left(4 k^{2}-1\right)}{1440} .
\end{aligned}
$$

After simplification, we obtain

$$
A^{2}(k-3)(-2 k-1)=15 .
$$

For $k \geq 3$, the expression in the left-hand side above is negative or zero, which is a contradiction. If $k=2$, then $A^{2}=3$, which contradicts the fact that $A \in \mathbb{Q}$. Therefore there are no rational coefficients $a, b, c, d, A$ and $B$ such that (3.21) is fulfilled, which implies that $\operatorname{deg} \varphi=1$. 
Now, we have

$$
S_{a, b}^{k}\left(a_{1} x+a_{0}\right)=e_{1} f(x)+e_{0} \quad \text { and } \quad S_{c, d}^{l}\left(b_{1} x+b_{0}\right)=e_{1} g(x)+e_{0},
$$

where $0 \neq e_{1}, e_{0} \in \mathbb{Q}$. Further, we have $\operatorname{deg} f=k+1$ and $\operatorname{deg} g=l+1$.

In view of the assumptions on $k$ and $l$, it follows that the standard pair $(f, g)$ cannot be of the second kind, and with the exception of the case $(k, l)=$ $(3,5)$, of the fifth kind either.

If it is of the first kind, then one of the polynomials $S_{a, b}^{k}\left(a_{1} x+a_{0}\right)$ and $S_{c, d}^{l}\left(b_{1} x+b_{0}\right)$ is of the form $e_{1} x^{q}+e_{0}$ with $q \geq 3$. This is impossible by Lemma 2.4 .

If $(f, g)$ is a standard pair of the third or fourth kind, we then have $S_{c, d}^{l}\left(b_{1} x+b_{0}\right)=e_{1} D_{\nu}(x, \delta)+e_{0}$ with $\nu=l+1 \geq 5$ and $\delta \in \mathbb{Q}^{*}$, which contradicts Lemma 2.5 or $k=2, l=3$. In this case Theorem 1.4 gives an effective finiteness result.

Now returning to the special case $(k, l)=(3,5)$, by using formula $(2.10)$ for $k=3$ it is easy to see that $S_{a, b}^{3}\left(c_{1} c+c_{0}\right)=e_{1}\left(3 x^{4}-4 x^{3}\right)+e_{0}$ is impossible, see the proof of Lemma 2.4.

\section{ACKNOWLEDGEMENTS}

The authors are grateful to the referee for her/his careful reading and helpful remarks.

The research was supported in part by the Hungarian Academy of Sciences, by the OTKA grants K75566, K100339, NK101680, NK104208 and by the TÁMOP 4.2.1./B-09/1/KONV-2010-0007 project implemented through the New Hungary Development Plan cofinanced by the European Social Fund and the European Regional Development Fund. Dijana Kreso was supported by the Austrian Science Fund (FWF): W1230-N13 and NAWI Graz.

\section{REFERENCES}

[1] A. Bazsó, Á. Pintér and H. M. Srivastava, A refinement of Faulhaber's Theorem concerning sums of powers of natural numbers, Appl. Math. Lett. 25 (2012), 486-489.

[2] Y. F. Bilu and R. F. Tichy, The Diophantine equation $f(x)=g(y)$, Acta Arith. 95 (2000), 261-288.

[3] Y. F. Bilu, B. Brindza, P. Kirschenhofer, Á. Pintér and R. F. Tichy, Diophantine equations and Bernoulli polynomials (with an Appendix by A. Schinzel), Compositio Math. 131 (2002), 173-188.

[4] J. Brillhart, On the Euler and Bernoulli polynomials, J. Reine Angew. Math. 234 (1969), 45-64.

[5] B. Brindza, On S-integral solutions of the equation $y^{m}=f(x)$, Acta Math. Hungar. 44 (1984), 133-139.

[6] Á. Pintér and Cs. Rakaczki, On the zeros of shifted Bernoulli polynomials, Appl. Math. Comput. 187 (2007), 379-383.

[7] H. Rademacher, Topics in Analityc Number Theory, Springer-Verlag, Berlin, 1973. 
[8] Cs. Rakaczki, On some generalizations of the Diophantine equation $s\left(1^{k}+2^{k}+\cdots+\right.$ $\left.x^{k}\right)+r=d y^{n}$ Acta Arith. 151 (2012), 201-216

[9] J. J. Schäffer, The equation $1^{p}+2^{p}+3^{p}+\cdots+n^{p}=m^{q}$, Acta Math. 95 (1956), $155-189$.

A. Bazsó

Institute of Mathematics

MTA-DE Research Group "Equations, functions and curves"

Hungarian Academy of Science

University of Debrecen

H-4010 Debrecen, P.O. Box 12

Hungary

E-mail: bazsoa@science.unideb.hu

D. Kreso

Institut für Mathematik (A)

Technische Universität Graz

Steyrergasse 30, 8010 Graz

Austria

E-mail: kreso@math.tugraz.at

F. Luca

Mathematical Center UNAM

UNAM Ap. Postal 61-3 (Xangari)

CP 58 089, Morelia, Michoacán

Mexico

E-mail: fluca@matmor.unam.mx

Á. Pintér

Institute of Mathematics

MTA-DE Research Group "Equations, functions and curves"

Hungarian Academy of Science

University of Debrecen

H-4010 Debrecen, P.O. Box 12

Hungary

E-mail: apinter@science.unideb.hu

Received: 7.11.2011.

Revised: 27.12.2011. 